
JNM
J Neurogastroenterol Motil, Vol. 25 No. 2 April, 2019
pISSN: 2093-0879 elSSN: 2093-0887
https://doi.org/10.5056/jnm18150

\title{
Opioid Treatment and Excessive Alcohol Consumption Are Associated With Esophagogastric Junction Disorders
}

\author{
Valeria Schindler, ${ }^{1}$ Daniel Runggaldier, ${ }^{1,2}$ Amanda Bianca, ${ }^{1}$ Anton S Becker, ${ }^{3}$ Fritz Murray, ${ }^{1}$ Edoardo Savarino, ${ }^{4}$ and Daniel Pohl ${ }^{1 *}$ \\ ${ }^{1}$ Division of Gastroenterology and Hepatology, University Hospital Zurich, Switzerland; ${ }^{2}$ Department of Otorhinolaryngology, University \\ Hospital of Zurich, Switzerland; ${ }^{3}$ Institute of Diagnostic and Interventional Radiology, University Hospital Zurich, Switzerland; and ${ }^{4}$ Department \\ of Surgery, Oncology and Gastroenterology, University of Padua, Italy
}

\section{Background/Aims}

The influence of external factors such as opioids and alcohol has been extensively investigated for various segments of the gastrointestinal tract. However, the association between their use and the development of esophagogastric junction outflow obstruction disorders (EGJOODs) is unknown. Therefore, the aim of this study is to analyze prevalence and clinical relevance of opioids and alcohol intake in patients with EGJOODs.

\section{Methods}

In this single-center, retrospective study, we reviewed clinical and pharmacological data of 375 consecutive patients who had undergone high resolution impedance manometry for EGJOODs. EGJOODs were classified according to the Chicago classification version 3.0 and to recently published normal values for test meals. Demographics, manometric data, and symptoms were compared between different groups using Pearson's chi-squared test, Fisher's exact test, and multivariate analysis. A $P<0.05$ was considered significant.

\section{Results}

EGJOOD was found in $30.7 \%(115 / 375)$ of all analyzed patients. The prevalence of opioids $(14.8 \%$ vs $4.2 \%, P=0.026)$ was significantly higher in patients with EGJOODs compared to patients without EGJOODs. Additionally, excessive alcohol consumption $(12.2 \%$ vs $3.5 \%, P=0.011)$ was associated with EGJOODs. Excessive alcohol consumption was especially frequent in the nonachalasia esophagogastric junction outflow obstruction subgroup (16.2\%) and opioid use in the achalasia type III subgroup (20.0\%).

\section{Conclusions}

We found a significant association between EGJOODs and opioid as well as excessive alcohol consumption. This underlines the importance of detailed history taking regarding medication and ethanol consumption in patients with dysphagia. Further prospective studies on mechanisms undelaying esophagogastric junction dysfunction due to opioids or alcohol are warranted.

(J Neurogastroenterol Motil 2019;25:205-211)

Key Words

Alcohol drinking; Analgesics, Opioids; Esophageal achalasia; Esophagogastric junction; Manometry

Received: September 6, 2018 Revised: December 6, 2018 Accepted: January 8, 2019

(.) This is an Open Access article distributed under the terms of the Creative Commons Attribution Non-Commercial License (http://creativecommons. org/licenses/by-nc/4.0) which permits unrestricted non-commercial use, distribution, and reproduction in any medium, provided the original work is properly cited.

*Correspondence: Daniel Pohl, MD

Division of Gastroenterology and Hepatology, University Hospital Zurich, Raemistr. 100, 8091 Zurich, Switzerland

Tel: +41 4425511 11, Fax: +41 4425545 91, E-mail: daniel.pohl@usz.ch

Valeria Schindler and Daniel Runggaldier contributed equally to this work. 


\section{Introduction}

Esophagogastric junction outflow obstruction disorders (EGJOODs) are a group of esophageal motility disorders often characterized by an impairment of food intake and symptoms such as dysphagia, odynophagia, regurgitations, and weight loss. Hence, affected individuals often suffer a significantly reduced quality of life. ${ }^{1}$ EGJOODs are characterized by an elevated integrated relaxation pressure (IRP) during high-resolution impedance manometry (HRIM) and are further subdivided into achalasia type I-III and esophagogastric junction outflow obstruction (EGJOO) according to Chicago classification (CC; version 3.0). This categorization depends on the level of impairment of peristalsis and the esophageal pressurization pattern upon swallowing (Fig. 1). ${ }^{2}$ Current standard testing protocols of esophageal physiology incorporate single lowvolume water swallows that frequently fail to detect a significant quantity of esophageal motility disorders and usually do not evoke symptoms. Additional testing such as multiple rapid swallows and, more recently, meal testing offer a more physiologic insight into patient deglutition dynamics and better symptom association (Fig. $1 \mathrm{E}$ and $1 \mathrm{~F}){ }^{3}$ While the diagnostic process and further classification into achalasia type I-III and EGJOO is well established, the underlying etiology and pathophysiological processes are not fully understood. Esophageal peristalsis and lower esophageal sphincter
(LES) relaxation are processes primarily controlled by ganglionic cells of the myenteric plexus, which can either induce esophageal smooth muscle cell contraction or relaxation, depending on the neurotransmitter released. ${ }^{4,5}$ Hence, neurodegenerative, autoimmune and viral processes causing an imbalance between the excitatory and inhibitory transmission, eg, by the loss of ganglion cells, are thought to be involved in the pathogenesis of EGJOODs. ${ }^{6-10}$ The classical EGJOO ( $\mathrm{CC}$ v3.0), which is characterized by elevated IRP but preserved peristalsis, could also be triggered by other factors such as hiatal hernia, impaired crural relaxation, or a mechanical obstruction, potentially caused by fibrotic stricture. ${ }^{11}$ Furthermore, the expression of opioid receptors was shown in the esophageal body and LES, suggesting a potential influence of opioids on esophageal motility and esophagogastric junction (EGJ) relaxation. ${ }^{12-15}$ Indeed a higher IRP as well as lower distal latency on esophageal pressure topography have been shown. ${ }^{12}$ Moreover, in patients with dysphagia and chronic opioid use an impaired LES relaxation and simultaneous esophageal waves were noticed. ${ }^{16}$ However, a deeper knowledge on opioid induced EGJOOD is still scarce.

Not only opioids but also alcohol seems to affect the upper gastrointestinal tract. The most known effect of excessive alcohol consumption is the development of esophageal varices as a consequence of liver damage. While there is accordance with the increased prevalence of gastroesophageal reflux in alcoholics, there are inconsistent data of the impact on the LES. Reduced pressure as well as
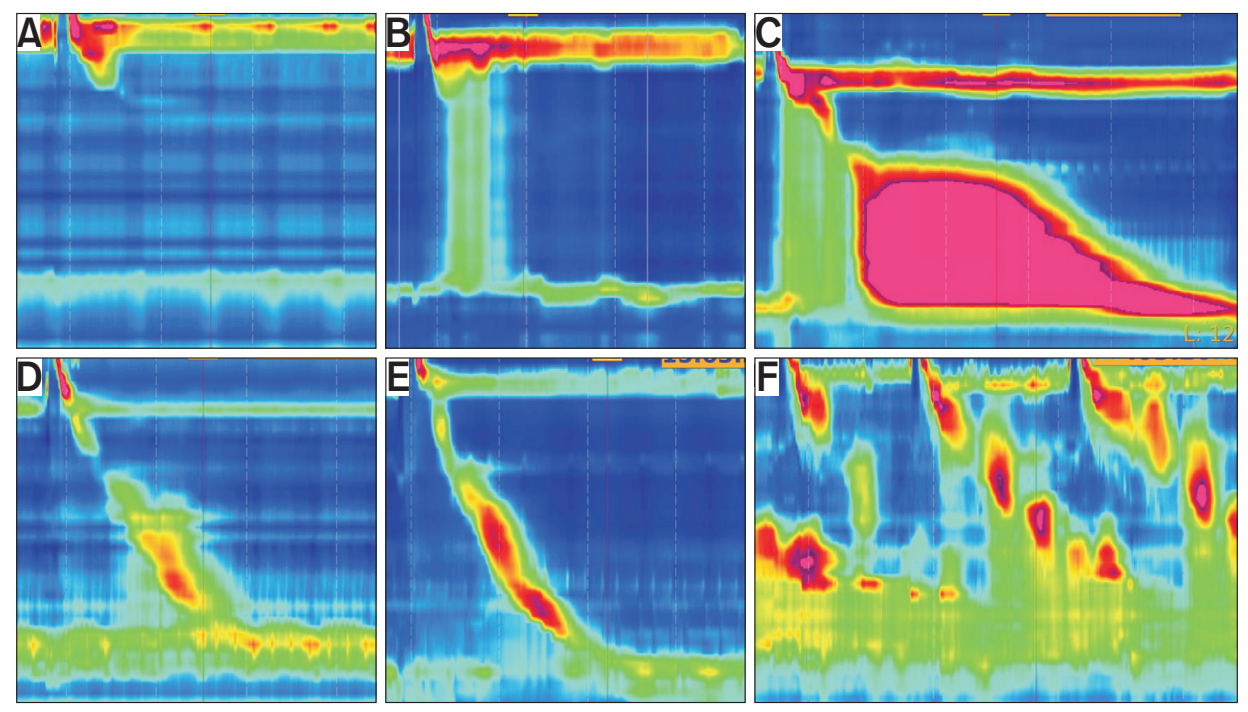

Figure 1. Clouse plots of high-resolution impedance manometry of the different esophagogastric junction outflow obstruction (EGJOO) disorders with achalasia type I (A), type II (B), type III (C), and EGJOO Chicago classification version 3.0 (D). Clouse plot of a single water swallow of a patient with an EGJOO in the standardized test meal (E). Clouse plot of the same patient during a rice test meal (F). The y-axis corresponds to the distance from proximal to distal esophagus, the x-axis constitutes the time. The local pressure at a given time-point is color coded (low pressure $=$ blue, intermediate pressure $=$ green/yellow, and high pressure $=\mathrm{red} /$ purple). 
a hypertensive LES have been described in conjunction with alcohol. ${ }^{17-19}$ Moreover, alcohol has recently been linked with esophageal dysmotility, $^{20,21}$ but its role in the development of EGJOODs is still unclear. Therefore, the aim of this study is to analyze the prevalence and clinical relevance in terms of symptomatic esophageal dysfunction of opioids and alcohol intake in patients diagnosed with EGJOODs.

\section{Materials and Methods}

We reviewed data of 375 consecutive patients for alcohol consumption (sporadic, regular, and excessive) and opioid use who had undergone HRIM after being referred to our tertiary referral center for symptoms of esophageal dysfunction between 2015 and 2017. All patients underwent endoscopic evaluation including esophageal biopsies to rule out structural etiologies prior to HRIM. Inclusion criteria were age older than 18 years and signed general informed consent. Ethics approval was obtained for the analysis (BASEC-Nr. 2017-00930).

\section{High-resolution Impedance Manometry}

HRIM was performed in a standardized protocol. ${ }^{22}$ The catheter was inserted nasally in seated position, following local anesthesia with lidocaine gel of the nostrils. Resting pressures of the lower and upper esophageal sphincters were measured after an adaption period of approximately 2 minutes. Then, 10 single swallows, each with $10 \mathrm{~mL}$ of water, were performed in semi-upright position. Afterwards, patients were asked to eat a test meal consisting of cooked plain white rice (200 g portion) at liberty, but within a maximum period of 8 minutes, as previously discussed. ${ }^{3,23}$ Before and after the test meal, a rapid drink challenge of $200 \mathrm{~mL}$ water using a drinking straw was performed.

\section{Esophagogastric Junction Outflow Obstruction Classification}

HRIM findings were classified according to $\mathrm{CC}$ v3.0 based on 10 single water swallows. EGJOODs were subdivided in achalasia type I-III and EGJOO (Fig. 1). ${ }^{2}$ Patients not classifying for EGJOO according to $\mathrm{CC}$ v3.0 were reevaluated for EGJOO according to recently published indicative HRIM values ${ }^{3}$ during the test meal (Fig. 1D-F).

\section{Opioids and Alcohol}

Data on alcohol consumption and opioid use were collected from medical records. Alcohol consumption (sporadic and/or regu- lar) was assessed by patient history. Excessive alcohol consumption was defined as $>2$ standard units ( $24 \mathrm{~g}$ alcohol) daily for men and $>1$ standard unit (12 $\mathrm{g}$ alcohol) for women according the Federal Center for Health, Germany. Opioid use was assessed by patients' medication lists. In all opioid users morphine equivalent doses (MED) were calculated.

\section{Statistical Methods}

Statistical analysis was performed with $\mathrm{R}$ version 3.2.0 ( $\mathrm{R}$ Foundation for Statistical Computing, Vienna, Austria). Numerical data are presented as mean \pm standard deviation (SD) for normally distributed data, and as median and interquartile range (IQR) in not normally distributed data. Normal distribution was assessed visually with normal quantile-quantile plots. For 2-group comparison of categorical data, Pearson's chi-squared test, Fisher's exact test, and odds ratios (OR) were used. The multivariate analysis was based on the type of EGJOOD, opioid, and alcohol consumption as well as demographics. For the analysis of MED in relation to EGJOOD Kruskal Wallis test was used. A $P$-value $<0.05$ was considered statistically significant. All tests were 2 -tailed.

\section{Results}

\section{Patient Characteristics and Demographics}

A total of 375 consecutive patients with no evidence of organic disease at upper endoscopy were considered. The prevalence of EGJOODs among all analyzed patients was 30.7\% (115/375). Major motility disorders other than EGJOODs were diagnosed in $20.3 \%$ (75/375) of patients and minor motility disorders in $6.6 \%$ $(25 / 375)$ of cases. In the group of EGJOODs the prevalence of achalasia type I was $2.6 \%$ (3/115), type II $24.3 \%$ (28/115), type III 8.7\% (10/115), EGJOO (CC v3.0) 30.4\% (35/115), and EGJOO (standardized test meal [STM]) 33.9\% (39/115). Demographics of the group with EGJOODs were significantly different compared to the non-EGJOOD group regarding age (64 years vs 53 years, $P<0.001)$, but not regarding gender (46.9\% vs $52.7 \%, P=$ 0.362). Indication for HRIM (multiple symptoms accepted) were dysphagia (45.4\%), upper abdominal discomfort (40.3\%), acid regurgitation $(37.1 \%)$, heartburn $(33.9 \%)$, and regurgitation of liquid and solid foods (18.4\%).

\section{Association of Esophagogastric Junction Outflow Obstruction Disorders With Opioids}

Indications for opioid consumption were musculoskeletal pain 
syndromes in 20/28 patients $(71.4 \%)$, codeine use for cough in 2 $(7.1 \%)$, methadone maintenance treatment in $2(7.1 \%)$, loperamide use for chronic diarrhea in 1 (3.6\%), and unknown indications in 3 patients (10.7\%). All included opioid types are listed in Figure 2.

Opioid consumption was significantly more prevalent in patients with EGJOODs (14.7\% [17/115]) as compared to patients without EGJOODs (4.2\% [11/260]) (OR, 3.9; 95\% CI, 1.88.9) (Fig. 3A). Within the group of opioid consumers, patients with EGJOODs consumed significantly higher doses of morphine $(P=0.023)$ (Fig. 4). Prevalence of EGJOODs in patients with

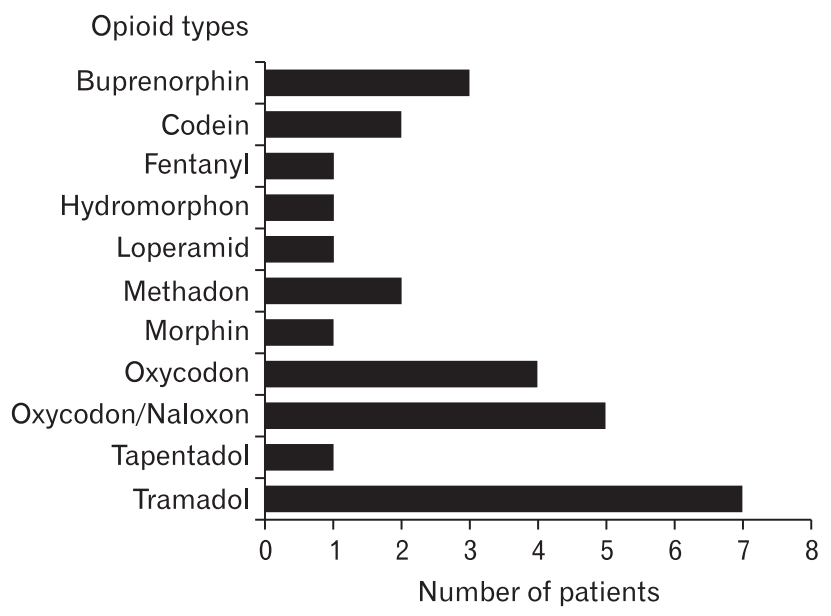

Figure 2. Number of patients using opioids.

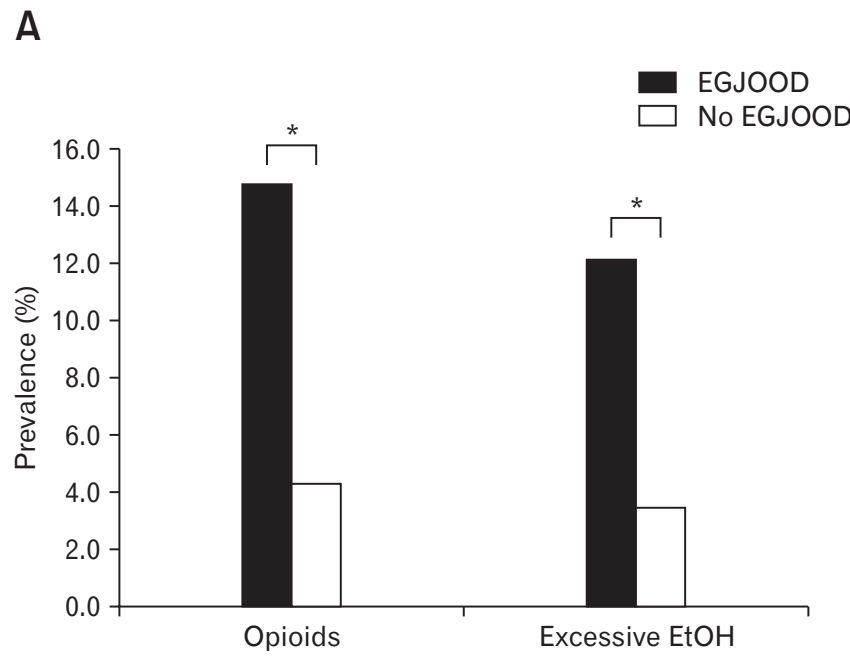

MEDs higher than $100 \mathrm{mg}$ per day was $100.0 \%$ (7/7) while it was $47.6 \%(10 / 21)$ in patients consuming less than $100 \mathrm{mg}$.

Patients with achalasia type III had the highest prevalence of opioid usage (20.0\% [2/10]), followed by EGJOO (water swallow, CC v3.0; 17.1\%, 6/35), EGJOO (STM 15.4\% [6/39]), achalasia type II (10.7\% [3/28]), and achalasia type I (0.0\% [0/3]) (Fig. $3 \mathrm{~B})$. No association of opioid consumption with other major and minor motility disorders was found.

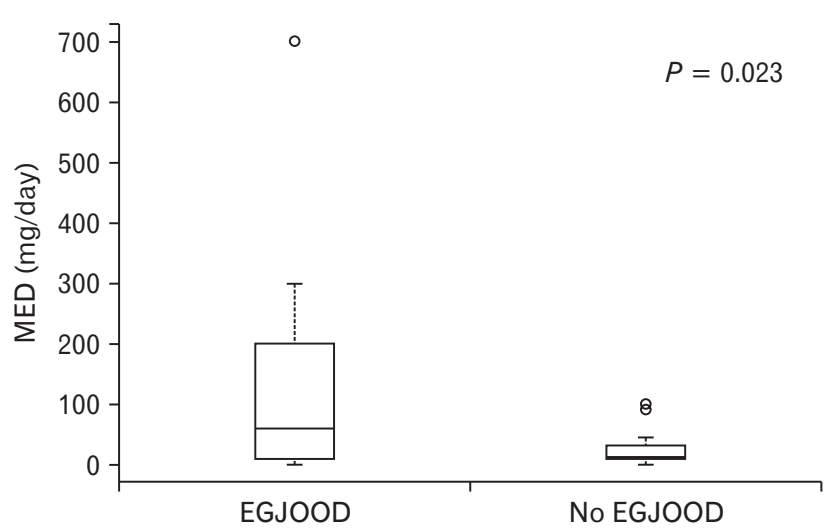

Figure 4. Morphine equivalent doses (MED) in opioid consumers in relation to esophagogastric junction outflow obstruction disorder (EGJOOD). The Kruskal-Wallis test was used for statistical analysis.

B

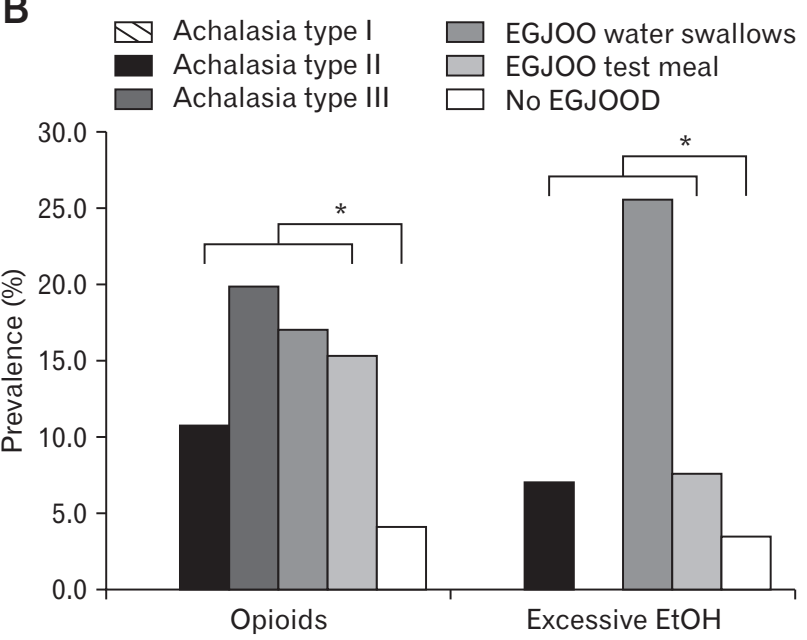

Figure 3. Prevalence of opioid and excessive alcohol consumption in patients according to esophagogastric outflow obstruction disorder (EGJOOD). (A) Prevalence of opioid consumption and excessive alcohol (EtOH) consumption in patients with EGJOOD versus patients without EGJOOD. (B) Prevalence of opioid consumption and excessive EtOH consumption in patients with achalasia type I-III, esophagogastric outflow obstruction (EGJOO) Chicago classification version 3.0, EGJOO in test meals and patients without any EGJOOD. ${ }^{*} P<0.01$, generalized linear model was used for the multivariate analysis. 
A

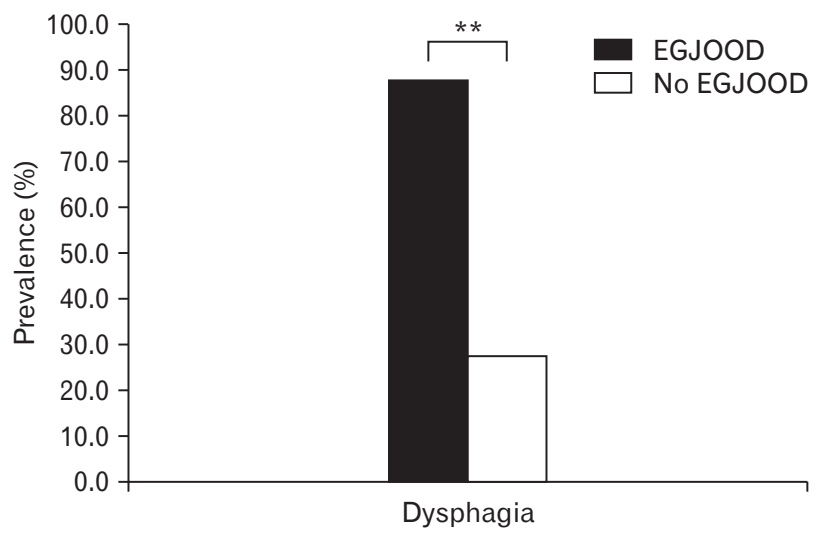

B

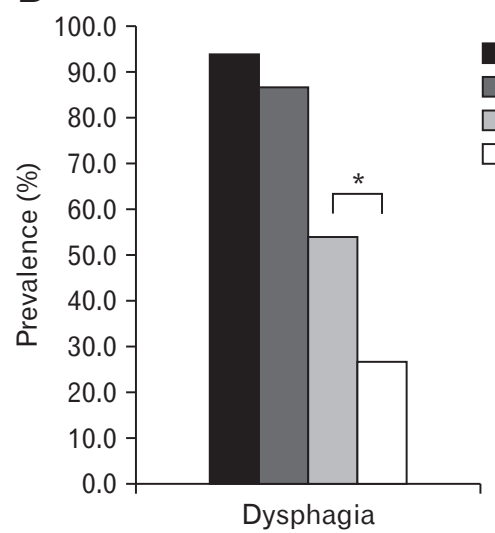

EGJOOD + opioid EGJOOD - opioid No EGJOOD + opioid No EGJOOD - opioid

Figure 5. Patients with dysphagia according to esophagogastric outflow obstruction disorder (EGJOOD). (A) Prevalence of dysphagia in patients with EGJOOD vs patients without EGJOOD. (B) Patients with and without EGJOOD and/or opioids reporting dysphagia. *P $<0.1,{ }^{*} P<0.001$ (Pearson's chi-squared test).

\section{Association of Esophagogastric Junction Outflow Obstruction Disorders With Alcohol Consumption}

Of all patients diagnosed with EGJOODs, $12.2 \%$ (14/115) were found to excessively consume alcohol versus only $3.5 \%$ (9/260) in the group of patients without an EGJOOD (OR, 3.9; 95\% CI, 1.6-9.5) (Fig. 3). In a subgroup analysis we also found a significantly different prevalence of EGJOODs according to the amount of alcohol consumption $(P=0.006)$. In excessive alcohol consumer the prevalence of EGJOODs was $60.9 \%$ (14/23), in occasional alcohol consumers $25.8 \%$ (34/132), in patients with rare alcohol consumption 25.0\% (15/60) and in abstinent patients 32.5\% (52/160).

In the subgroup analysis of EGJOODs (achalasia type I to III vs non-achalasia EGJOO, according to CC v3.0 and STM), we found excessive alcohol consumption in only $4.8 \%$ (2/41) of achalasia patients but $16.2 \%(12 / 74)$ of patients with an EGJOO itself $(P$ $<0.001)$.

\section{Effect of Opioids and Excessive Alcohol Consumption on Upper Gastrointestinal Symptoms}

Dysphagia and regurgitation were found significantly more often in patients with EGJOODs (OR, 18.5; 95\% CI, 10.2-35.7 and OR, 23.0; 95\% CI, 11.8-48.9, respectively) (Fig. 5A). In contrast, other symptoms such as heartburn, upper abdominal discomfort, chronic cough, and cervical globus sensation were predominantly found in patients without EGJOODs. Interestingly, a subgroup analysis showed a trend towards a higher prevalence of dysphagia in the group of non EGJOODs patients if those patients were treated with opioids (Fig. 5B). No other symptoms were influenced by concurrent opioid usage. Excessive alcohol consumption, however, did not influence any of the described symptoms.

\section{Discussion}

In this single-center, retrospective study, we reviewed the data of 375 consecutive patients with unremarkable endoscopy who had undergone HRIM for symptoms of esophageal dysfunction. In order to increase the diagnostic sensitivity for EGJOODs, STMs were used additionally to the single water swallows as published recently. ${ }^{23}$ In particular, we investigated the association between esophageal abnormalities and alcohol as well as opioids use. Opioid usage has recently become a global epidemic, most pronounced in North America. ${ }^{24-26}$ Similarly, the rate of excessive alcohol abuse has increased significantly in many parts of the world. ${ }^{27-29}$ Hence, the understanding of adverse effects of opioids and alcohol abuse with potential effects on esophageal motility is of evermore importance. Our analysis revealed several findings: first, we found that the prevalence of opioid usage was significantly higher in patients with EGJ outflow obstruction compared to the group without an EGJOOD. Interestingly we also found in the context that within the group of opioid consumers, patients with EGJOODs consumed significantly higher doses of opioids suggesting a dose dependent opioid effect. It has to be pointed out that all opioids were taken for indications unrelated to EGJOODs. Interestingly, patients diagnosed with achalasia type III had the highest prevalence of opioid consumption, followed by EGJOO. In contrast, we neither found an association of major motility disorders such as hypercontractile esophagus nor minor motility disorders with opioid usage. 
Second, our analysis revealed that dysphagia, a cardinal symptom of achalasia, tended to be more frequent in patients without EGJOOD that were treated with opioids. This indicates that in addition to the well-known adverse effects like constipation and nausea, opioids might also cause esophageal symptoms even in the absence of a quantifiable EGJOODs, suggesting either subclinical outlet dysfunction or an influence on the sensory component. In agreement to the above mentioned investigation, a recent study analyzing the prevalence of opioid usage in a group of patients diagnosed with achalasia found that opioid usage was most common in type III achalasia. ${ }^{13}$ Furthermore, a study comparing patients on and off opioid medication revealed that opioid usage within 24 hours of HRIM was associated with a higher frequency of EGJOO as well as type III achalasia.

Third, we identified a significant association between excessive alcohol consumption and EGJOODs, especially in patients diagnosed with non-achalasia EGJOO. After the exclusion of external compression of the EGJ, the underlying etiology and pathophysiology of the EGJOO is poorly understood: a recent study identified a decreased immune-reactivity of endothelial nitric oxide synthase in the esophageal myenteric plexus, additionally to a significantly decreased serotonin-induced relaxation of the LES in ethanol-fed rats. ${ }^{20}$ In line with the limited animal data, we speculate that excessive alcohol consumption might lead to EGJOO by interfering with the nitric oxide mediated relaxation of the LES. A possible other mechanism might be mediated via an increased rate of gastroesophageal reflux, which in turn leads to inflammation and consecutive stiffening of the EGJ. The latter hypothesis would also explain why alcohol abuse is only associated with the EGJOO itself, but with no other motility disorders of the esophageal body such as achalasia. Other alcohol induced causes might be neuropathy or impairment of contractile proteins, as previously hypothesized for bowel motility disorders. ${ }^{20}$

We recognize that our study does have certain limitations. The retrospective nature of the study is connected to a risk of confounding factors despite the usage of a multivariate modelling to mitigate this problem. Even though the study was conducted at a university hospital with patients from a wide geographical area, it was a single center study in a tertiary center which further limits the generalizability. Moreover, we do not have any HRIM data after cessation of opioid treatment or excessive alcohol consumption which hinders us to directly compare patients "on" and "off" opioids/excessive alcohol. Hence, to reveal the exact pathophysiologic mechanisms of opioid and ethanol induced EGJOODs, more basic and clinical research, including multicenter studies, are needed.
In summary, our data support the hypothesis of heterogeneous pathophysiologic processes and etiology of EGJOODs, which might not only be triggered by autoimmune disease as proposed for achalasia type I and II. ${ }^{4,9,30}$ In accordance to the presented data, we suggest an important role and dose dependent effect of opioids and alcohol in the development of EGJOODs. Especially the high prevalence of opioid treatment and excessive ethanol consumption in patients diagnosed with achalasia type III and EGJOO may be the result of ganglionic dysfunction or imbalance of the proposed excitatory and inhibitory neurotransmission of the LES region. Since some of these proposed effects are potentially reversible, further studies are warranted investigating the replacement or stoppage of the identified triggers as a feasible treatment option in patients with type III achalasia or classical outflow obstruction. In addition, our results emphasize the importance of a detailed history taking regarding medication and alcohol consumption in patients presenting with suspected esophageal dysfunction and dysphagia.

\section{Financial support: None.}

\section{Conflicts of interest: None.}

Author contributions: Valeria Schindler and Daniel Runggaldier: designed the study, collected and analyzed the data, and statistical analysis, and wrote the manuscript; Amanda Bianca: collected and analyzed the data and edited the manuscript; Anton S Becker: analyzed data, statistical analysis and edited the manuscript; Fritz Murray: edited the manuscript; Edoardo Savarino: edited and approved the manuscript; and Daniel Pohl: study concept, designed the study and edited and approved the manuscript.

\section{References}

1. Tuason J, Inoue H. Current status of achalasia management: a review on diagnosis and treatment. J Gastroenterol 2017;52:401-406.

2. Kahrilas PJ, Bredenoord AJ, Fox M, et al. The Chicago classification of esophageal motility disorders, v3.0. Neurogastroenterol Motil 2015;27:160-174.

3. Hollenstein M, Thwaites P, Bütikofer S, et al. Pharyngeal swallowing and oesophageal motility during a solid meal test: a prospective study in healthy volunteers and patients with major motility disorders. Lancet Gastroenterol Hepatol 2017;2:644-653.

4. Furuzawa-Carballeda J, Torres-Landa S, Valdovinos MÁ, Coss-Adame E, Martín Del Campo LA, Torres-Villalobos G. New insights into the pathophysiology of achalasia and implications for future treatment. World J Gastroenterol 2016;22:7892-7907.

5. Park W, Vaezi MF. Etiology and pathogenesis of achalasia: the current 
understanding. Am J Gastroenterol 2005;100:1404-1414.

6. Boeckxstaens GE. Achalasia: virus-induced euthanasia of neurons? Am J Gastroenterol 2008;103:1610-1612.

7. Clark SB, Rice TW, Tubbs RR, Richter JE, Goldblum JR. The nature of the myenteric infiltrate in achalasia: an immunohistochemical analysis. Am J Surg Pathol 2000;24:1153-1158.

8. Kornizky Y, Heller I, Isakov A, Shapira I, Topilsky M. Dysphagia with multiple autoimmune disease. Clin Rheumatol 2000;19:321-323.

9. Runggaldier D, Fried M, Pohl D. Recurrent episodes of esophageal candidiasis without dysphagia post-Guillain-Barré syndrome: an unusual presentation of achalasia. BMJ Case Rep 2017 Published Online First:15 Oct 2017. doi: 10.1136/bcr-2017-221751.

10. Booy JD, Takata J, Tomlinson G, Urbach DR. The prevalence of autoimmune disease in patients with esophageal achalasia. Dis Esophagus 2012;25:209-213.

11. van Hoeij FB, Smout AJ, Bredenoord AJ. Characterization of idiopathic esophagogastric junction outflow obstruction. Neurogastroenterol Motil 2015;27:1310-1316.

12. Ratuapli SK, Crowell MD, DiBaise JK, et al. Opioid-induced esophageal dysfunction (OIED) in patients on chronic opioids. Am J Gastroenterol 2015;110:979-984.

13. Ravi K, Murray JA, Geno DM, Katzka DA. Achalasia and chronic opiate use: innocent bystanders or associated conditions? Dis Esophagus 2016;29:15-21.

14. Liu XB, Liu JF. Expression of dopamine receptors in human lower esophageal sphincter. J Gastroenterol Hepatol 2012;27:945-950.

15. Rattan S, Goyal RK. Identification and localization of opioid receptors in the opossum lower esophageal sphincter. J Pharmacol Exp Ther 1983;224:391-397.

16. Kraichely RE, Arora AS, Murray JA. Opiate-induced oesophageal dysmotility. Aliment Pharmacol Ther 2010;31:601-606.

17. Kaufman SE, Kaye MD. Induction of gastro-oesophageal reflux by alcohol. Gut 1978;19:336-338.

18. Keshavarzian A, Polepalle C, Iber FL, Durkin M. Esophageal motor disorder in alcoholics: result of alcoholism or withdrawal? Alcohol Clin Exp Res 1990;14:561-567.

19. Grande L, Monforte R, Ros E, et al. High amplitude contractions in the middle third of the oesophagus: a manometric marker of chronic alcoholism? Gut 1996;38:655-662.

20. Yazir Y, Tugay M, Utkan Z, Utkan T. Effects of chronic ethanol consumption on rat upper gastrointestinal system: functional and histologic findings. Alcohol 2012;46:649-655.

21. Bujanda L. The effects of alcohol consumption upon the gastrointestinal tract. Am J Gastroenterol 2000;95:3374-3382.

22. Gyawali CP, de Bortoli N, Clarke J, et al. Indications and interpretation of esophageal function testing. Ann N Y Acad Sci 2018;1434:239-253.

23. Ang D, Misselwitz B, Hollenstein M, et al. Diagnostic yield of highresolution manometry with a solid test meal for clinically relevant, symptomatic oesophageal motility disorders: serial diagnostic study. Lancet Gastroenterol Hepatol 2017;2:654-661.

24. Berterame S, Erthal J, Thomas J, et al. Use of and barriers to access to opioid analgesics: a worldwide, regional, and national study. Lancet 2016;387:1644-1656.

25. Fox M, Menne D, Stutz B, Fried M, Schwizer W. The effects of tegaserod on oesophageal function and bolus transport in healthy volunteers: studies using concurrent high-resolution manometry and videofluoroscopy. Aliment Pharmacol Ther 2006;24:1017-1027.

26. National Institute on Drug Abuse. Opioid overdose crisis [revised January 2019]. Available from URL: https:/www.drugabuse.gov/drugsabuse/opioids/opioid-overdose-crisis (accessed 3 March 2019).

27. Grant BF, Chou SP, Saha TD, et al. Prevalence of 12-month alcohol use, high-risk drinking, and DSM-IV alcohol use disorder in the united states, 2001-2002 to 2012-2013: results from the national epidemiologic survey on alcohol and related conditions. JAMA Psychiatry 2017;74:911923.

28. Pimpin L, Cortez-Pinto H, Negro F, et al. Burden of liver disease in Europe: epidemiology and analysis of risk factors to identify prevention policies. J Hepatol 2018;69:718-735.

29. Babor TF. Out of africa: three threads tying the alcohol industry to an emerging alcohol epidemic. J Stud Alcohol Drugs 2018;79:157-158.

30. Furuzawa-Carballeda J, Aguilar-León D, Gamboa-Domínguez A, et al. Achalasia--an autoimmune inflammatory disease: a cross-sectional study. J Immunol Res 2015;2015:729217. 